\title{
Antibiotic Resistance Profile of Staphylococci Isolated From Hospital Out-Patients in Accident and Emergency Unit
}

\author{
Ojo, S. K. S.*, Onyechi, T. O. ${ }^{1}$, Esumeh, F. I. ${ }^{2}$ \\ *Department of Microbiology, Federal University, Oye-Ekiti, Ekiti State, Nigeria \\ ${ }^{1}$ Department of Biological Sciences, Novena University, Ogume, Delta State, Nigeria. \\ ${ }^{2}$ Department of Microbiology, Ambrose Alli University, Ekpoma, Edo State, Nigeria.
}

\begin{abstract}
S$. aureus, a major pathogenic strain in man with wide antibiotic resistance profile vis-ă-vis the emerging resistance pattern encountered in coagulase-negative staphylococci to penicillin family and fluoroquinolones. Fifty (50) wounds and burns out-patients samples undergoing injury dressing in General Hospital was identified, characterized and evaluated for resistant pattern yielding 21(42\%) Staphylococcus spp. with $18(86 \%)$ coagulase-positive strains and 3(14\%) coagulase-negative staphylococci, 16(76\%) mannitol fermenters and 5(24\%) non-mannitol fermenters. The $\beta$-lactamase assay resulted in $6(28.6 \%) \beta$-lactamase producers $S$. aureus and $1(4.8 \%) \beta$-lactamase producer CoNS, while $14(67 \%)$ are non- $\beta$-lactamase producers. The resistance pattern of $\beta$-lactamase and non- $\beta$-lactamase producing staphylococci showed that the highest resistance pattern for Staphylococcus aureus group was observed with ampiclox, amoxicillin, ceftriaxone 15(83.3\%), while in CoNS group, resistance was observed in cefuroxime, streptomycin, amoxicillin, ampiclox and gentamicin representing 2(66.7\%) each. The MAR index for Staphylococcus spp. isolated ranges from 0.2 to 1.0 with $76.1 \%$ isolates showing resistance to at least 6 antibiotics. Therefore, resistance pattern of normal skin flora to regularly prescribed antibiotics is becoming alarming and demands health safety consciousness/awareness.
\end{abstract}

Keywords: wounds and burns, S. aureus, coagulase-negative staphylococci, $\beta$-lactamase, antibiotics

\section{Introduction}

Staphylococci are a group of ubiquitous microorganisms important in human and animal infections (Ako-Nai et al., 2005). In the past ten years, staphylococci have re-emerged as predominant organisms causing infections in hospital setting. They are responsible for various medical problems, which includes: skin and soft tissues infections, surgical site infections, endocarditis, hospital-acquired bacteraemia (Gamal et al., 2010). According to Ombui et al. (2000), strains of S. aureus resistant to multiple antibiotics including methicillin and gentamicin were increasingly responsible for outbreaks of hospital infections in countries around the world during the late 1970s and early 1980s. Coagulase-negative staphylococci (CoNS) are mainly important component of the normal skin flora, which has also emerged as an important pathogen in foreign body infections. Most developed countries have reported an increase in colonization and infection in hospitalized patients by CoNS while there are scanty data on infections caused by CoNS in developing countries (Bashir et al., 2007; Akinjogunla and Enabulele, 2010).

Since the introduction of antimicrobials, bacteria have developed mechanisms for resisting the effects of antibiotics. The levels of antibiotic resistant infections in the developing world have increased steadily in the last few decades as a result of combination of microbial characteristics and the selective pressure of antimicrobial used. The emergence of multidrug resistance in Gram-positive bacteria (pneumococci, enterococci and staphylococci) is particularly important development. Perhaps the pathogen of greatest concern are the staphylococci, because of its intrinsic virulence, its ability to cause an array of life threatening conditions, and its capacity to adapt to different environmental conditions (Bashir et al., 2007; Akinjogunla and Enabulele, 2010).

The prevalence of antibiotic resistance worldwide due to antibiotic overuse or underuse (misuse) both within medicine and veterinary, has led to the failure of even newly discovered antibiotics, thus giving impetus to this study. This study intends to evaluate the resistance pattern of different antibiotics exposed among wounds and burns out-patients within the accident and emergency unit of the hospital or as outpatient using over the counter (OTC)self prescription.

\section{Materials And Methods}

Sample Collection and Processing: Fifty (50) clinical samples were obtained from out-patients undergoing injury dressing of wounds and burns at the accident and emergency unit of the General Hospital, Kwale, Delta State, Nigeria. Wounds and burns exudates were obtained from the infected site of each patient with sterile cotton swabs and applied to freshly prepared slants of Nutrient agar (NA) and Mannitol Salt agar 
(MSA) (Oxoid). The cultures were then transferred to the laboratory where they were incubated at $37^{\circ} \mathrm{C}$ for $24 \mathrm{hr}$.

2.1. Isolation and Identification of isolates: Colonies growing on slants were streaked on the freshly prepared plates of MSA and incubated at $35^{\circ} \mathrm{C}$. Primary characterization was based on Gram staining, morphological and cultural characteristic on different media including NA, fermentation on MSA, catalase and coagulase (tube) test. The ability of each $S$. aureus and CoNS isolates to elaborate penicillinase was evaluated using the method as described by Ako-Nai et al. (2005).

2.2. $\beta$-lactamase Assay: This test was carried our as described by Ako-Nai et al. (2005). Strips of starch paper measuring $4 \mathrm{~cm} \times 7 \mathrm{~cm}$ were cut and sterilized with $70 \%$ ethanol. These strips were then soaked for $10 \mathrm{mins}$ in a solution of benzyl penicillin dissolved in phosphate buffer containing $10^{5}$ units. They were spread over an area of 2 to $3 \mathrm{~mm}$. Each test paper was then used to test two (2) organisms at a time with the inocula placed at least $2 \mathrm{~cm}$ apart. The Petri dishes were then incubated for $30 \mathrm{mins}$ at $37^{\circ} \mathrm{C}$ after which the plate was flooded with Grams iodine solution. This was immediately drained off. This caused the starch paper to turn uniformly black within 30seconds of application. Colonies with decolourized zones thereafter were indicative of $\beta$-lactamase production. Results were read within 5mins as black background tends to decolourize, making interpretations more difficult.

2.3. Determination of Antibiotic Resistance Profile: Wounds and burns isolates were subjected to antibiotic screening by disk diffusion method as described by CLSI (2008). Inocula were prepared by diluting overnight cultures in sterile $\mathrm{NaCl}(0.9 \%)$ suspension and then marched with the McFarland turbidity index. Bacterial suspensions were then plated on to MHA and the commercially available antibiotic discs were placed on lawn of culture and the plates incubated overnight at $37^{\circ} \mathrm{C}$. Sensitivity, intermediate and resistance were determined by the zone of complete growth inhibition around each disk according to reference standards. Reference type S. aureus strain (ATCC 25923) was used as positive control. The percentage resistance was calculated using the formula: $\mathrm{PR}=\mathrm{a} / \mathrm{b} \times 100$, where 'PR' was percentage resistance, ' $a$ ' was the number of resistant isolates and ' $b$ ' was the number of isolates tested with the antibiotic. The percentage sensitivity was calculated using the formula: $\mathrm{PS}=\mathrm{c} / \mathrm{d}$ x 100, where 'PS' was percentage sensitivity, 'c' was the number of sensitive isolates and ' $\mathrm{d}$ ' was the number of isolates tested with the antibiotic. The percentage intermediate was calculated using the formula: $\mathrm{PI}=\mathrm{e} / \mathrm{f}$ x 100, where 'PI' was percentage intermediate, 'e' was the number of intermediate isolates and ' $\mathrm{f}$ ' was the number of isolates tested with the antibiotic.

2.4. Determination of Multiple Antibiotic Resistance (MAR) Index: The MAR index was determined using the formula MAR $=\mathrm{x} / \mathrm{y}$, where $\mathrm{x}$ was the number of antibiotics to which test isolates displayed resistance and $\mathrm{y}$ is the total number of antibiotics to which test organism has been evaluated for sensitivity. The percentage resistance for MAR index was calculated as PR' $=\alpha / \Upsilon \times 100$, where PR' was percentage resistance for MAR index, ' $\alpha$ ' was the number of test isolates of same species with multiple antibiotic resistance and ' $Y$ ' was total number of test isolates of same species.

2.5. Statistical Analysis of Results: Frequencies and percentages were calculated for study variables. Chi-square $\left(\mathrm{X}^{2}\right)$ test was used to calculate probabilities and determine significance. A p-value of more than or equal to 0.05 was considered to be statistically significant $(\mathrm{p} \geq 0.05)$, while $\mathrm{p}$-value less than 0.05 was considered to be statistically not significant.

\section{Results}

Fifty (50) wounds and burns exudates collected from hospital patients undergoing dressing yielded $21(42 \%)$ isolates. $14(66.7 \%)$ staphylococci isolates were recovered from the wound samples while 7(33.3\%) isolates from burns samples. 18(86\%) isolates indicate Coagulase-positive $S$. aureus and $3(14 \%)$ are Coagulasenegative staphylococci (CoNS). 16(76\%) isolates showed fermentation on mannitol while 5(24\%) showed no fermentation (Table 1). The $\beta$-lactamase assay carried out on the hospital isolates revealed presence of $\beta$ lactamase producing species in $7(33 \%)$ isolates and $14(67 \%)$ non- $\beta$-lactamase producing species (Table 2). The susceptibility pattern of isolated staphylococci as compared to CLSI (2008) standards is as indicated in Table 3.

Staphylococcus aureus group ( $\beta$-lactamase and non- $\beta$-lactamase producer) was slightly sensitive to cotrimoxazole, streptomycin and gentamicin $(27.8 \%)$ while coagulase-negative staphylococci ( $\beta$-lactamase and non- $\beta$-lactamase producer) group had the highest sensitivity pattern of $66.7 \%$ on Co-trimoxazole and erythromycin. The highest resistance pattern for Staphylococcus aureus group was observed with ampiclox, amoxicillin and ceftriaxone $15(83.3 \%$ ) each followed by erythromycin $14(77.7 \%)$, ciprofloxacin $13(72.2 \%)$ and cefuroxime, pefloxacin and gentamicin $12(66.7 \%)$ each. However, the highest resistance pattern in CoNS group 
was observed in cefuroxime, streptomycin, amoxicillin, ampiclox and gentamicin representing $2(66.7 \%)$ each.

Table 4, showed the antibiogram pattern of multiple antibiotics resistance. $5(23.8 \%)$ isolates were resistant to the 10 tested antibiotics, 4(19.0\%) isolates resistant to 7 antibiotics. The MAR index calculated (Table5), indicate that the antibiotic resistant Staphylococcus spp. ranged from 0.2 to 1.0. The result showed that $76.1 \%$ Staphylococcus spp. were resistant to at least 6 antibiotics. Thus indicating a multiple drug resistance pattern in the isolates, this could eventually become community acquired infection.

Table1. Biochemical characterization and identification of wounds and burns isolates from hospital patients in accident and emergency unit.

\begin{tabular}{lcl}
\hline Biochemical test & No of staphylococcal isolates (\%) \\
\hline & Positive & Negative/others \\
\cline { 2 - 3 } Gram staining & $21(42 \%)$ & $29(58 \%)$ \\
Catalase & 21 & - \\
Coagulase & $18(86 \%)$ & $3(14 \%)$ \\
Mannitol fermentation & $16(76 \%)$ & $5(24 \%)$ \\
\hline
\end{tabular}

Table2. Characterization and occurrence of $\beta$-lactamase and non- $\beta$-lactamase producing $S$. aureus and CoNS isolated from wounds and burns patients

\begin{tabular}{llll} 
Isolate & No of & No $(\%)$ of $\beta L$ & No/(\%) of Non- $\beta I$ \\
& Occurrence & producer & producer \\
\hline S. aureus & 18 & $6(28.6 \%)$ & $12(57.1 \%)$ \\
CoNS & 3 & $1(4.8)$ & $2(9.5 \%)$ \\
\hline
\end{tabular}

Table3. Antibiotic susceptibility profile on isolated Staphylococcus spp. from wounds and burns hospital patients

\begin{tabular}{|c|c|c|c|c|c|c|}
\hline \multirow[t]{3}{*}{ Antibiotics } & \multicolumn{3}{|c|}{ Staphylococcus aureus group $(\mathrm{n}=18)$} & \multicolumn{3}{|c|}{ CoNS group $(\mathrm{n}=3)$} \\
\hline & \multicolumn{6}{|c|}{ Sensitive Intermediate Resistant } \\
\hline & No $(\%)$ & $\mathrm{No}(\%)$ & $\mathrm{No}(\%)$ & No(\%) & $\mathrm{No}(\%)$ & $\mathrm{Ne}(\%)$ \\
\hline Co-trimoxazole (Cot) & $5(27.8)$ & $2(11.1)$ & $11(61.1)$ & $2(66.7)$ & $0(0.0)$ & $1(33.3)$ \\
\hline Erythromycin (E) & $4(22.2)$ & $0(0.0)$ & $14(77.7)$ & $2(66.7)$ & $(0.0)$ & $1(33.3)$ \\
\hline Pefloxacin (PEF) & $3(16.7)$ & $3(16.7)$ & $12(66.7)$ & $1(33.3)$ & $1(33.3)$ & $1(33.3)$ \\
\hline Cefuroxime (Cex) & \multicolumn{2}{|c|}{$1(5.6)$} & $5(27.8)$ & $12(66.7)$ & \multicolumn{2}{|r|}{$1(33.3)$} \\
\hline \multicolumn{7}{|l|}{$2(66.7)$} \\
\hline Ceftriaxone (Cef) & $0(0.0)$ & $3(16.7)$ & $15(83.3)$ & $0(0.0)$ & $2(66.7)$ & $1(33.3)$ \\
\hline Ciprofloxacin (CPX) & $1(5.6)$ & $4(22.2)$ & $13(72.2)$ & $1(33.3)$ & $1(33.3)$ & $1(33.3)$ \\
\hline Streptomycin (S) & $5(27.8)$ & $3(16.7)$ & $10(55.6)$ & $1(33.3)$ & $0(0.0)$ & $2(66.7)$ \\
\hline Amoxicillin (AM) & $3(16.7)$ & $0(0.0)$ & $15(83.3)$ & $1(33.3)$ & $0(0.0)$ & $2(66.7)$ \\
\hline Ampiclox (APX) & $3(16.7)$ & $0(0.0)$ & $15(83.3)$ & $1(33.3)$ & $0(0.0)$ & $2(66.7)$ \\
\hline Gentamicin (CN) & $5(27.8)$ & $1(5.6)$ & $12(66.7)$ & $1(33.3)$ & $0(0.0)$ & $2(66.7)$ \\
\hline
\end{tabular}




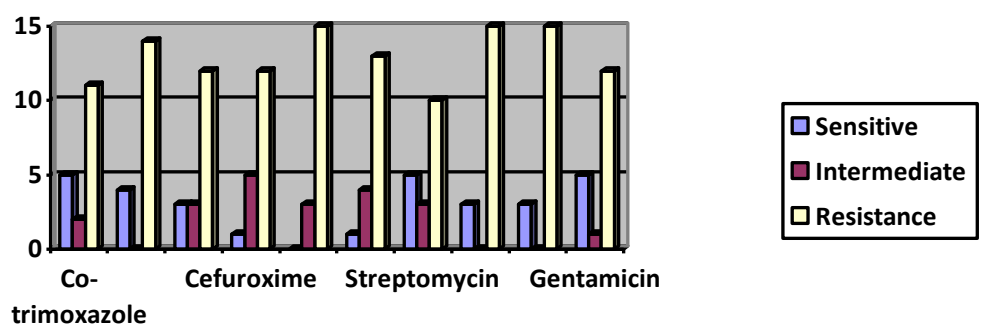

Figure1. Susceptibility pattern of S. aureus ( $\beta$-lactamase and non- $\beta$-lactamase producing) isolates
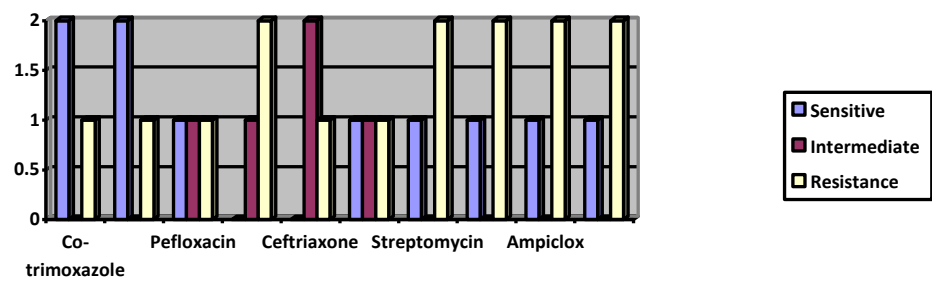

Figure2. Susceptibility pattern of CoNS ( $\beta$-lactamase and non- $\beta$-lactamase producing) isolates

Table4. Antibiogram patterns to multiple antibiotic resistance Staphylococci isolates

\begin{tabular}{clc}
\hline No of isolates & Pattems of Antibiotic resistance & Multiplicity \\
\hline 2 & Cex, S, AM, CN & 4 \\
1 & Cot, E, Cex, Cef, AM, APX & 6 \\
2 & Cot, E, PEF, Cef, CPX, AM & 6 \\
1 & E, PEF, Cef, CPX, S, APX, CN & 7 \\
1 & E, PEF, Cex, Cef, CPX, S, AM, APX & 8 \\
1 & E, Cex, Cef, S, AM, APX, CN & 7 \\
1 & E, PEF, Cef, CPX, S, AM, APX & 7 \\
5 & Cot, E, PEF, Cex, Cef, CPX, S, AM, APX, CN & 10 \\
1 & Cot, E, PEF, Cex, Cef, CPX, AM, APX & 8 \\
2 & Cot, E, PEF, Cex, Cef, CPX, AM, APX, CN & 9 \\
1 & Cot, CPX, Cex, Cef, S, AM, APX & 7 \\
3 & APX, CN & 2 \\
\hline
\end{tabular}

Table5. Multiple Antibiotic Resistance (MAR) Index of Staphylococci isolated from hospital patients

\begin{tabular}{cll}
\hline $\begin{array}{l}\text { No of resistant } \\
\text { Isolates (\%) }\end{array}$ & \multicolumn{2}{l}{ No of antibiotics MAR Index } \\
\hline $4(19.0)$ & 7 & 0.7 \\
$2(9.5)$ & 9 & 0.9 \\
$2(9.5)$ & 8 & 0.8 \\
$5(23.8)$ & 10 & 1.0 \\
$314.3)$ & 6 & 0.6 \\
$3(14.3)$ & 2 & 0.2 \\
$2(9.5)$ & 4 & 0.4 \\
\hline
\end{tabular}

\section{Discussion}

The study was conducted to determine the susceptibility and resistance profile of the isolates from accident and emergency unit of the hospital. The isolation of CoNS and $\beta$-lactamase producing CoNS in this study was in agreement with Akinjogunla and Enabulele (2010), who isolated 9(42.9\%) CoNS, Akinkunmi and Lamikanra (2010) isolated 118(40.3\%) CoNS while Ako-Nai et al. (2005) isolated 52(31\%) CoNS strains and $26(50 \%)$ of $\beta$-lactamase producing CoNS. The rate of isolation from various sites of infection as reported by various authors becomes paramount in creating hospital/health awareness of the possible spread either as a nosocomial infection or community-acquired infection. This study also isolated $\beta$-lactamase producing 
S. aureus, which conform to earlier studies by Ako-Nai et al. (2005), Akinjogunla and Enabulele (2010) and Bashir et al. (2007).

The susceptibility pattern of Staphylococcus spp. to various antibiotics has been reported. In this study, $S$. aureus group ( $\beta$-lactamase and non- $\beta$-lactamase producing) showed a low sensitivity rate pattern with the highest in co-trimoxazole, streptomycin and gentamicin $(27.8 \%$ each) while the high resistance of S. aureus and $\beta$-lactamase producing $S$. aureus encountered with ampiclox, amoxicillin, ceftriaxone, cefuroxime, erythromycin, pefloxacin and ciprofloxacin and gentamicin in this study was similar to earlier reports. Adegoke and Okoh (2011) reported a high erythromycin resistance, 51\% erythromycin resistance by Bashir et al.(2007), 95\% in Yameen et al. (2010), while Ako-Nai et al. (2005) reported (54.3\%) resistance to erythromycin, amoxicillin (64.9\% \& 86.8\%). 87\% ciprofloxacin resistance by Al-Ruaily and Khalil (2011) \& $80 \%$ by Yameen et al. (2010). Akinjogunla and Enabulele (2010) reported 50\% resistance in amoxicillin. The high gentamicin resistance observed from this study against $S$. aureus isolates do not conform to similar studies by Maithem and Intisar (2012), Adegoke and Okoh (2011), Yameen et al. (2010), Al-Ruaily and Khalil (2011), and Ako-Nai et al. (2005) who observed low resistance. CoNS and $\beta$-lactamase producing CoNS equally revealed high resistance to ampiclox, amoxicillin, cefuroxime, streptomycin and gentamicin. This was in accordance with Akinjogunla and Enabulele (2010) who reported 57.1\% and 52.4\% resistance to streptomycin and amoxicillin respectively, 69.2\% amoxicillin resistance in Ako-Nai et al. (2005). However, gentamicin resistance to CoNS group in this study was not in agreement with Ako-Nai et al. (2005) and Akinkunmi and Lamikanra (2010) who observed low resistance. The low resistance of fluoroquinolones to CoNS in this study was also reflected by Akinkunmi and Lamikanra (2010) and Akinjogunla and Enabulele (2010). It was observed that the high resistance rate in fluoroquinolones and gentamicin against $S$. aureus isolates from the study area was indicative of self-prescription (OTC) by patients, and veterinary abuse. The MAR index from this study was within range as observed by Akinjogunla and Enabulele (2010).

\section{Conclusion}

The exceedingly increases and emergence of multidrug resistance pathogens especially $\beta$-lactamase producing Staphylococcus spp. in the developing countries can be attributed to indiscriminate use of antibiotics, complex socio-economic, behavioural antecedents and dissemination of drug-resistant pathogens in human medicine and more importantly in the veterinary since humans have a direct association either as pet or for consumption. Thus, $\beta$-lactamase producing $S$. aureus and CoNS have posed a great threat to clinical practice in the administration of antibiotics in this part of the continent and needs urgent public health attention.

\section{Acknowledgement}

We sincerely appreciate Dr. Omumu and his medical team at the General Hospital, Kwale, Delta State, for their medical advice and support during collection of clinical specimens and Prof. Ogi Okwumabua of Wisconsin University, U.S.A for his advice and donation of CLSI manuals.

\section{References}

[1]. A.A. Adegoke and A.I. Okoh. The in vitro effect of vancomycin on multidrug resistant Staphylococcus aureus from hospital currency notes. African Journal of Microbiology Research 5(14), 2011, 1881-1887.

[2]. O.J., Akinjogunla and I.O., Enabulele. Virulence factors, plasmid profiling and curing analysis of multi-drug resistant Staphylococcus aureus and coagulase negative Staphylococcus spp. isolated from patients with acute otitis media. Journal of American Science 6(11), 2010, 1022-1033

[3]. A.K., Ako-Nai, F.M., Adeyemi, O.A., Aboderin, O.O., Kassim. Antibiotic resistance profile of Staphylococci from clinical sources recovered from patients. African Journal of Biotechnology 4(8), 2005, 816-822.

[4]. M.A., Al-Ruaily and O.M., Khalil. Detection of (mecA) gene in methicillin resistant Staphylococcus aureus (MRSA) at Prince A/Rhman Sidery Hospital, Al-Jouf, Saudi Arabia. Journal of Medical Genetics and Genomics 3(3), 2011, 41-45.

[5]. A, Bashir, Y.M., Talata, and J., Nayar. Antibiotic resistance profile: isolation and characterization of clinical isolates of staphylococci from patients with community-acquired skin infections. Pak. J. Pharm. Sci. 20(4), 2007, 295-299

[6]. H.F., Chambers. The changing epidemiology of Staphylococcus aureus. Emerging Infectious Diseases 7(2), 2001, 178-82

[7]. Clinical Laboratory Standards Institute (CLSI). Performance standards for antimicrobial susceptibility testing; $18^{\text {th }}$ informational supplement. M100-S18. 28(1), 2008. Wayne, Pennsylvania, U.S.A.

[8]. F.M.G., Gamal, F.A., Abd El-Ghafar, A.A.E., Ramadan, S.H. Zeinab. Epidemiology and antimicrobial resistance of staphylococci isolated from different infectious diseases. Brazillian Journal of Microbiology 41(2), 2010. doi: 10.1590/S151783822010000200012

[9]. A.A., Maithem and G.H. Intisar. Study of plasmid profile, susceptibility patterns of clinical Staphylococcus aureus isolated from patients with otitis media in Basrah. Journal of Basrah Researches (Sciences) 38(1.A), 2012: 79-89.

[10]. Minnesota Department of Health. Staphylococcus aureus - Infectious Disease Epidemiology, Prevention and Control 2010. Retrieved from www.health.state.mn.us. June 30, 2011

[11]. J.N. Ombui, A.M. Kimotho and J.G. Nduhiu. Antimicrobial resistance patterns and plasmid profiles of Staphylococcus aureus isolated from milk and meat. East African Medical Journal 77(9), 2000, 463-467

[12]. M.A. Yahmeen, H. Nasim, N. Akhtar, S. Iram, I. Javed and A. Hameed. Antibiotic susceptibility profile of methicillin-resistant staphylococci isolated from nasal samples of hospitalized patients. African Journal of Microbiology Research 4(3), 2010, 204-209 